\section{Bei Rhinitis auch an die Augen denken!}

Eü ir die Behandlung der allergischen Rhinitis empfehlen die Leitlinien der ARIA-Initiative (Allergic Rhinitis and its Impact on Asthma) für alle Schwergrade intranasale Glukortikoide. Fluticasonfuroat $\left(\right.$ Avamys $\left.{ }^{\circledR}\right)$ habe dabei von allen verfügbaren Substanzen die höchste Affinität zum Glukokortikoidrezeptor und eine besonders lange Verweildauer im Nasengewebe, erklärte Dr. Bettina Hauswald, Dresden. Das gewährleiste eine 24-Stunden-Wirkung. Außerdem werde der Wirkstoff schon in der Nase vollständig abgebaut (sehr niedrige Bioverfügbarkeit von $0,5 \%$ ), so dass keine systemischen Nebenwirkungen zu befürchten seien. Fluticasonfuroat verbessert zudem nicht nur die nasalen Symptome der all- ergischen Rhinitis, sondern auch die okulären.

Eine aktuelle Metaanalyse von 35 plazebokontrollierten Studien hat ergeben, dass nur Fluticasonfuroat bei saisonaler allergischer Rhinitis eine konsistent positive Wirkung aufdie Augensymptome hat. Bei anderen intranasalen Glukortikoiden hingegen waren die Effekte widersprüchlich und inkonsistent, berichtete Dr. Oliver Pfaar, Wiesbaden. Weitere Studien mit Fluticasonfuroat haben gezeigt, dass sich neben der Symptomatik auch alle untersuchten Domänen der Lebensqualität der Patienten mit allergischer Rhinitis unter der Therapie signifikant und konsistent verbesserten.

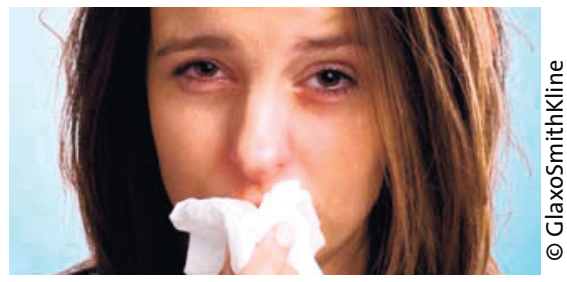

Etwa drei Viertel der Rhinits-Patienten leiden auch unter Augensymptomen.

Die Behandlung mit Fluticasonfuroat ist dabei auch unter Kostenaspekten effektiv: Anfang Februar 2010 wurde der Preis um 25\% reduziert, was den Verordnungsspielraum vergrößert.

aam

Pressegespräch „Start der Allergiesaison: Nasen- und Augensymptome jetzt schon wirksam und kostengünstig mit Avamys ${ }^{\circledR}$ behandeln“. München, 23. Februar 2010. Veranstalter: GlaxoSmithKline, München

\title{
Sicher durch den Sommer bei Insektengiftallergie
}

P ro Jahr sterben in Europa rund 200 Menschen an den Folgen eines Bienen- oder Wespenstichs. Trotzdem wird die Insektengiftallergie nicht ausreichend ernst genommen - nur etwa jeder fünfte Betroffene sucht Hilfe beim Arzt. Um das öffentliche Bewusstsein für die Problematik zu schärfen, hat das Unternehmen ALK-Abelló in Deutschland, Österreich und der Schweiz in Zusammenarbeit mit einem länderübergreifenden medizinischen Expertenbeirat die „Initiative Insektengift-Allergie“ ins Leben gerufen.

Unter dem Motto "Sicher durch den Sommer" geht es bei der neuen Initiative vor allem darum, den Betroffenen die Gefahren vor Augen zu führen und ihnen zu zeigen, wie sie sich vor den lebensbedrohlichen Folgen einer schweren allergischen Reaktion schützen können. Auf der Website der Initiative www.initiative-insektengift.de sind umfassende Informationen über Vorbeugung, Anzeichen, Behandlung und richtiges Verhalten im Notfall sowie Tipps und Hilfestellungen abrufbar. Über den Menüpunkt „Insektengift Allergie“ gelangt der Nutzer zu den Rubri-

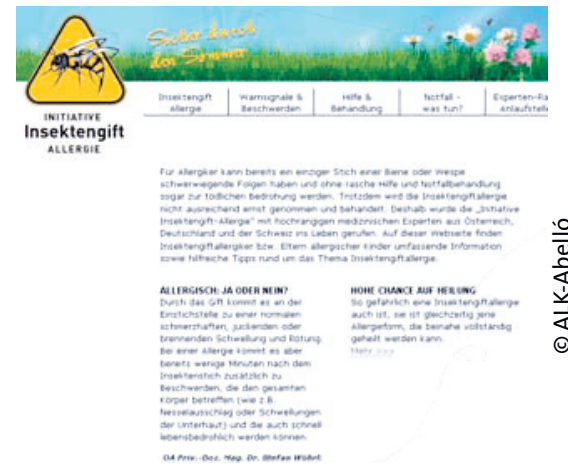

Unter www.initiative-insektengift.de können sich Betroffene laiengerecht über die Gefahren einer Insektengiftallergie informieren.

ken „Die wichtigsten Insekten“ und „Lebensgefährliche Allergien“. Dort findet er eine Tabelle mit den Flugsaisons verschiedener Hautflügler und erfährt, was nach einem Stich im Körper passiert. Symptome und Schweregrade einer allergischen Reaktion sind unter „Warnsignale \& Beschwerden “ in leicht erfassbaren Schemata zusammengetragen. Wie laufen Diagnostik und Impfung beim Facharzt ab? Das beantwortet anschaulich bebildert „Hilfe \& Behand- lung“. Bei „Notfall - was tun“ wird erklärt, welche Erste-Hilfe-Maßnahmen zu beachten sind und was ein Notfallset ist. Ein Fünf-Fragen-Selbsttest und verschiedene Ratgeber zum Ausdrucken stehen unter "Service \& Downloads“ zur Verfügung. Die Website klärt insbesondere über die Bedeutung der spezifischen Immuntherapie auf, die noch zu wenig genutzt wird. Der Experte Prof. Dr. Bernhard Przybilla aus München betont: „Bei Mastozytose und Insektengiftallergie ist das Risiko für eine lebensbedrohliche Anaphylaxie besonders hoch - etwa $80 \%$ der allergischen Reaktionen sind hier schwer oder sehr schwer, das heißt, es kommt zum Allergieschock oder zum Herz-KreislaufStillstand. Auch diese Patienten können durch spezifische Immuntherapie vor erneuten Reaktionen geschützt werden."

Über die Patienteninformationen hinaus gibt es auch einen kleinen Bereich für Ärzte. Dort sind Materialien für das Patientenmanagement wie ein Patientenfragebogen und ein Notfallposter abrufbar. Alles in allem eine übersichtliche Seite, von der Betroffene und Behandler profitieren können.

Nach Informationen von ALK-Abelló, Hørsholm, Dänemark 